\title{
A comparison of Marchenko strategies for the treatment of internal multiples and their consequences for seismic imaging
}

\author{
Rodrigo S. Santos ${ }^{1,2}$, Marcelo S. Souza ${ }^{1,2}$, Daniel E. Revelo ${ }^{1,2}$, Reynam C. Pestana ${ }^{2}$, Diego F. Barrera ${ }^{1}$, Victor Koehne ${ }^{1}$ \\ ${ }^{1}$ SENAI/CIMATEC, ${ }^{2}$ UFBA
}

Copyright 2021, SBGf - Sociedade Brasileira de Geofísica.

This paper was prepared for presentation during the $17^{\text {th }}$ International Congress of the Brazilian Geophysical Society, held in Rio de Janeiro, Brazil, 8-11 November 2021.

Contents of this paper were reviewed by the Technical Committee of the $17^{\text {th }}$ International Congress of the Brazilian Geophysical Society and do not necessarily represent any position of the SBGf, its officers or members. Electronic reproduction or storage of any part of this paper for commercial purposes without the written consent of the Brazilian Geophysical Society is prohibited.

\begin{abstract}
Seismic imaging of the subsurface is normally made through (single-scattering) conventional techniques that treat the internal multiple reflections as noise. Recently, strategies to remove these migrated false events have been developed. Technologies such as source-receiver Marchenko redatuming with adaptive double-focusing method (DFM) and Marchenko multiple elimination by least-squares (LSMME) have been shown as very efficient methods to solve this problem. Both methods are based on the Marchenko equations and they can be used as a preprocessing step to generate seismic images without artifacts related to internal multiple events. In this work, we compare these methods and evaluate their success with numerical examples. Then, we demonstrate the robustness of the LSMME scheme removing internal multiples, when compared with DFM, albeit at a higher computational cost.
\end{abstract}

\section{Introduction}

Seismic processing steps such as velocity analysis and standard imaging in time and depth (Kirchhoff, RTM, etc) present limitations when there are internal multiples. The multiples are created when waves reflect more than once in the subsurface before reaching the receivers. However, standard imaging methods such as reversetime migration (RTM) or Kirchhoff time/depth migration, rely on the single-scattering assumption, i.e., recorded seismic data does not include multiples. Though these events have lower energy than the primary reflections, the single-scattering assumption can lead to the generation of strong artifacts in the seismic images. Some techniques have been proposed to mitigate the artifacts related to the internal multiples, whose traditional workflow consists of predicting the internal multiples and subtracting them from the acquired seismic data. However, with the recent use of the Marchenko equations, several alternatives have been presented for the treatment of this kind of information.

van der Neut and Wapenaar (2016) proposed a new method rewriting the coupled Marchenko equations. This scheme requires only a smooth velocity model to create time truncations. Zhang and Staring (2018) modified the scheme of van der Neut and Wapenaar (2016) and obtained the method called Marchenko multiple elimination (MME), which is a data-driven algorithm. Later, Zhang et al. (2019) used the work of Zhang and Staring (2018) to derive a scheme that eliminates internal multiple reflections and applies compensation for transmission losses contained in primary reflections. These results suggest that the MME scheme may be the best alternative to eliminate internal multiples when the seismic data has previously gone through a high-quality pre-processing stage, i.e., deghosting, removal of free-surface multiples and deconvolution with an estimated source wavelet. Zhang and Slob (2020b) have used a laboratory dataset to evaluate the performance of the MME, obtaining a dataset free of internal multiple reflections. Zhang and Slob (2019a) presented the first example of applying the MME on a field dataset from the Norwegian North Sea, which validated the capabilities of the MME scheme and showed that it can effectively eliminate internal multiples without model information or adaptive subtraction. Zhang and Slob (2020a) developed a fast implementation version that reduces the computational cost of the proposed scheme by one order of magnitude. Later, Santos et al. (2020b) showed that because the MME method is datadriven, it is possible to apply it in early seismic processing workflows and calculate NMO velocity fields by semblance picking free from the effects of internal multiples. This study demonstrates that internal multiple reflections have influence on the velocity analysis at early processing stages and that MME can be seen as a new tool in the conventional pre-processing workflows of seismic data. In the same year, Santos et al. (2020a) have proposed to formulate the application of the MME technique as a least-squares problem named of Marchenko multiples elimination by least-squares (LSMME) to avoid the stability issue related to the Neumann series expansion presented in the original formulation proposed by Zhang and Staring (2018).

Staring et al. (2018) developed an adaptive doublefocusing method (DFM) to remove the internal multiples caused by an overburden using source-receiver Marchenko redatuming. This method has the advantage to be more efficient in computational terms when compared with the multidimensional deconvolution (MDD) (van der Neut et al., 2011). To ensure the correct and complete removal of the internal multiples by their counter events is used an adaptive filter. This adds extra robustness to the method since the adaptive subtraction might be capable of correcting for the amplitudes mismatch. Additionally, the method of Staring et al. (2018) is less sensitive to data imperfections and sparse acquisition geometry, being an alternative method for source-receiver redatuming. On the other hand, it considers the physical medium above the redatuming level which leads to the remaining of some 
interactions with the overburden. Staring et al. (2018) show the success of the DFM using 2D synthetic data from the Santos basin offshore Brazil. Later, Staring and Wapenaar (2020) extended their work to three-dimensional data.

As shown by Staring et al. (2018), knowing the depth of the reservoir, it is possible to carry out a redatuming with the DFM for a region a little above it, thus allowing to obtain an image of the reservoir free of many of the multiple generated by layers above at a relatively low-cost. On the other hand, as shown in Santos et al. (2020a), the use of LSMME allows to obtain a multiples-free image of all orders and from all structures in the medium, but paying a much higher computational cost than the DFM. As in Wapenaar et al. (2021), which have discussed the different approaches to Marchenko redatuming, imaging and multiple elimination, using a common mathematical framework, in this paper, we compare the DFM with LSMME in the context of improving the image of a target region. The comparison covers the theory and the performance in a numerical example, where we show the advantages and disadvantages of both schemes and their influences on the migrated image.

\section{Theory}

\section{The LSMME scheme}

We follow Santos et al. (2020a) to give a brief overview of LSMME scheme in this section. To clarify our notation, the spatial coordinates are defined by their horizontal and depth components, for instance, $\mathbf{x}_{i}=\left(\mathbf{x}_{H}, z_{i}\right)$, where $\mathbf{x}_{H}$ are the horizontal coordinates and $z_{i}$ is the depth of an arbitrary boundary $\partial \mathbf{D}_{i}$, such that the surface acquisition $\partial \mathbf{D}_{0}$ will be defined by $\mathbf{x}_{0}=\left(\mathbf{x}_{H}, z_{0}\right)$. The acoustic impulse reflection response from a source at $\mathbf{x}_{0}$ (recorded by a pressure receiver at $\left.\mathbf{x}_{0}^{\prime}\right)$ is denoted as $R\left(\mathbf{x}_{0}^{\prime}, \mathbf{x}_{0}, t\right)$, where $t$ is the propagation time. To represent the reflection response, we use $\bar{R}\left(\mathbf{x}_{0}^{\prime}, \mathbf{x}_{0}, t\right)$. In practice, $R$ is obtained from deconvolution of $\bar{R}$ with the source time signature. The projected version of the revised Marchenko equations for the single-sided reflection response can be given by the following expressions (Zhang and Staring, 2018; Santos et al., 2020a):

$$
\begin{aligned}
& \left\{\begin{array}{l}
v_{m}^{+}\left(\mathbf{x}_{0}^{\prime}, \mathbf{x}_{0}^{\prime \prime}, t\right)=\left(\Theta_{\varepsilon}^{t_{2}-\varepsilon} \mathbf{R}^{*} v^{-}\right)\left(\mathbf{x}_{0}^{\prime}, \mathbf{x}_{0}^{\prime \prime}, t\right), \\
v^{-}\left(\mathbf{x}_{0}^{\prime}, \mathbf{x}_{0}^{\prime \prime}, t\right)=\left(\Theta_{\varepsilon}^{t_{2}-\varepsilon} \mathbf{R} \delta+\Theta_{\varepsilon}^{t_{2}-\varepsilon} \mathbf{R} v_{m}^{+}\right)\left(\mathbf{x}_{0}^{\prime}, \mathbf{x}_{0}^{\prime \prime}, t\right),
\end{array}\right. \\
& U^{-}\left(\mathbf{x}_{0}^{\prime \prime}, \mathbf{x}_{0}^{\prime}, t\right)=\left(\Theta_{t_{2}-\varepsilon}^{\infty} \mathbf{R} \delta+\Theta_{t_{2}-\varepsilon}^{\infty} \mathbf{R} v_{m}^{+}\right)\left(\mathbf{x}_{0}^{\prime \prime}, \mathbf{x}_{0}^{\prime}, t\right),
\end{aligned}
$$

where $U^{-}$are the projected versions of the upgoing Green function, and similarly, $v_{m}^{+}$and $v^{-}$from the focusing function. $v^{ \pm}$are also named by the down and upgoing filter functions, $t_{2}$ is the two-way traveltime of the acquisition surface $\partial \mathbf{D}_{0}$ and a fictitious reflector at horizon $\partial \mathbf{D}_{i}$. The $\Theta_{\varepsilon}^{t_{2}-\varepsilon}$ is a truncation operator to exclude values outside of the window $(\varepsilon, t-\varepsilon)$, where $\varepsilon$ is a positive value to account for the finite bandwidth. The operators $\mathbf{R}$ and $\mathbf{R}^{*}$ are defined as:

$$
\begin{aligned}
& \{\mathbf{R} P\}\left(\mathbf{x}^{\prime \prime}, \mathbf{x}^{\prime}, t\right)=\int_{\partial \mathbf{D}} d \mathbf{x} \int_{-\infty}^{+\infty} R\left(\mathbf{x}^{\prime}, \mathbf{x}, t^{\prime}\right) P\left(\mathbf{x}, \mathbf{x}^{\prime \prime}, t-t^{\prime}\right) d t^{\prime}, \\
& \left\{\mathbf{R}^{*} P\right\}\left(\mathbf{x}^{\prime \prime}, \mathbf{x}^{\prime}, t\right)=\int_{\partial \mathbf{D}} d \mathbf{x} \int_{-\infty}^{+\infty} R\left(\mathbf{x}^{\prime}, \mathbf{x},-t^{\prime}\right) P\left(\mathbf{x}, \mathbf{x}^{\prime \prime}, t-t^{\prime}\right) d t^{\prime} .
\end{aligned}
$$

Zhang and Slob (2019b) have shown that $U^{-}\left(\mathbf{x}_{0}^{\prime \prime}, \mathbf{x}_{0}^{\prime}, t\right)$ can be evaluated for each time instant $t_{2}$ in Equation 2. Then, if $U^{-}\left(\mathbf{x}_{0}^{\prime \prime}, \mathbf{x}_{0}^{\prime}, t\right)$ is convolved with the source wavelet, their value to $t_{2}$ can be collected to be stored in a new function $\bar{R}_{t}$, containing only primary reflections:

$$
\bar{R}_{t}\left(\mathbf{x}_{0}^{\prime \prime}, \mathbf{x}_{0}^{\prime}, t=t_{2}\right)=\bar{U}^{-}\left(\mathbf{x}_{0}^{\prime \prime}, \mathbf{x}_{0}^{\prime}, t_{2}\right) .
$$

The hat indicates that quantities have been convolved with the source wavelet. To evaluate $\bar{U}^{-}$it is first necessary to obtain $v_{m}^{+}$. Santos et al. (2020a) shows that $v_{m}^{+}$can be obtained if the Equation (1) is seen as an inverse problem, which can be solved using the least-squares scheme. So, starting with the affirmation presented in van der Neut and Wapenaar (2016), where is defined that $\{\mathbf{R} \delta\}\left(\mathbf{x}_{0}^{\prime \prime}, \mathbf{x}_{0}^{\prime}, t\right)=R\left(\mathbf{x}_{0}^{\prime \prime}, \mathbf{x}_{0}^{\prime}, t\right)$, we can rewrite these equations (1) in the following matricial form:

$$
\left[\begin{array}{c}
\mathbf{v}^{-} \\
\mathbf{v}_{m}^{+}
\end{array}\right]=\left[\begin{array}{l}
\Theta R+\Theta \mathbf{R} \mathbf{v}_{m}^{+} \\
\Theta \mathbf{R}^{*} \mathbf{v}^{-}
\end{array}\right] .
$$

Remembering that the least-squares method (LS) consists of obtaining an approximate solution $(\mathbf{x})$ to the problem in the form $\mathbf{A x}=\mathbf{y}$ by minimizing the sum of the squares of the residuals. After some algebraic manipulations in Equation (6), we can write:

$$
\underbrace{\left[\begin{array}{cc}
\mathbf{I} & -\Theta \mathbf{R} \\
-\Theta \mathbf{R}^{*} & \mathbf{I}
\end{array}\right]}_{\mathbf{A}} \underbrace{\left[\begin{array}{c}
\mathbf{v}^{-} \\
\mathbf{v}_{m}^{+}
\end{array}\right]}_{\mathbf{x}}=\underbrace{\left[\begin{array}{c}
\Theta R \\
\mathbf{0}
\end{array}\right]}_{\mathbf{y}} .
$$

By solving the system (7) with LS allows us to obtain $v_{m}^{+}$, which is necessary to compute $U^{-}$, we have the Marchenko multiple elimination schemes (see Equation (5)). To solve the linear system (7) we have applied the iterative method of Paige and Saunders (1982), which is based on a stable process. In this method, the elements of A are applied as operators, so it is not necessary to build the referred matrix. The solution of Marchenko multiple elimination based on least-squares is referred as LSMME.

\section{The Adaptive Double-Focusing method}

The adaptive double-focusing method, developed by Staring et al. (2018) is a source-receiver Marchenko redatuming based on two focusing steps: the obtaining of the downgoing focusing functions $f^{+}\left(\mathbf{x}_{0}, \mathbf{x}_{i}^{\prime}, t\right)$ that focus in a virtual source position in-depth, and the obtaining of the upgoing Green's function $G^{-}\left(\mathbf{x}_{i}^{\prime}, \mathbf{x}_{0}, t\right)$ in the position of a virtual receiver. The fundamental equation is based on the following multidimensional convolution:

$$
G^{-+}\left(\mathbf{x}_{i}, \mathbf{x}_{i}^{\prime}, t\right)=\int_{\partial D_{0}} G^{-}\left(\mathbf{x}_{i}, \mathbf{x}_{0}, t\right) * f^{+}\left(\mathbf{x}_{0}, \mathbf{x}_{i}^{\prime}, t\right) d^{2} \mathbf{x}_{0},
$$

where $G^{-+}$is the upgoing wavefield measured by a virtual receivers at $\mathbf{x}_{i}$ due to a virtual receiver at $\mathbf{x}_{i}^{\prime}$. This method creates virtual sources that propagate downward at the redatuming level and virtual receivers that register the upgoing wavefields (Staring et al., 2018). We obtain the wavefields $G^{-}$and $f^{+}$in Equation (8) by the following series:

$G^{-}\left(\mathbf{x}_{i}, \mathbf{x}_{0}, t\right)=\sum_{i=0}^{\infty} G_{i}^{-}\left(\mathbf{x}_{i}, \mathbf{x}_{0}, t\right)=\Psi\left(\mathbf{x}_{i}, \mathbf{x}_{0}, t\right) \mathbf{R} \sum_{i=0}^{\infty} \Omega^{i} f_{0}^{+}\left(\mathbf{x}_{0}, \mathbf{x}_{i}, t\right)$, 
and

$$
f^{+}\left(\mathbf{x}_{0}, \mathbf{x}_{i}^{\prime}, t\right)=\sum_{j=0}^{\infty} f_{j}^{+}\left(\mathbf{x}_{0}, \mathbf{x}_{i}^{\prime}, t\right)=\sum_{j=0}^{\infty} \Omega^{j} f_{0}^{+}\left(\mathbf{x}_{0}, \mathbf{x}_{i}^{\prime}, t\right),
$$

in which $\Omega$ represents the operator:

$$
\Omega=\theta \mathbf{R}^{*} \theta \mathbf{R},
$$

with $\theta$ being a Heaviside step function based on the oneway traveltime from the acquisition surface to the focal point and $\Psi$ a time window defined by $\Psi=1-\theta$. The variable $f_{0}^{+}$represents the direct part of the focusing function that is equal to the inverse direct arrival of the transmission response. The direct arrival of the transmitted wave can be obtained by seismic modeling using a smooth velocity field and applying a modeling method, e.g., finite-difference or an eikonal solver.

According to Staring et al. (2018), the initial wavefield $G_{0}^{-}=\Psi \mathbf{R} f_{0}^{+}$and $f_{0}^{+}$contain information of all arrivals and internal multiple reflections from the overburden, as well as, the updates of this wavefields, $G_{1}^{-}, G_{2}^{-}, f_{1}^{+}, f_{2}^{+}$, etc, contain only the multiples events, with opposite polarities and, initially, with incorrect amplitudes that adjust with the iterations. Since the main information of the multiple events is present in $f_{1}^{+}$and $G_{1}^{-}$, we can truncate the series at the first two terms. When truncating the series of Equations (9) and (10) and substituting in Equation (8) we have:

$$
\begin{aligned}
G^{-+}\left(\mathbf{x}_{i}, \mathbf{x}_{i}^{\prime}, t\right) & =\sum_{i=0}^{\infty} \sum_{j=0}^{\infty} \int_{\partial D_{0}} G_{i}^{-}\left(\mathbf{x}_{i}, \mathbf{x}_{0}, t\right) * f_{j}^{+}\left(\mathbf{x}_{0}, \mathbf{x}_{i}^{\prime}, t\right) d^{2} \mathbf{x}_{0} \\
\approx & \int_{\partial D_{0}} G_{0}^{-}\left(\mathbf{x}_{i}, \mathbf{x}_{0}, t\right) * f_{0}^{+}\left(\mathbf{x}_{0}, \mathbf{x}_{i}^{\prime}, t\right) d^{2} \mathbf{x}_{0} \\
& +\int_{\partial D_{0}} G_{1}^{-}\left(\mathbf{x}_{i}, \mathbf{x}_{0}, t\right) * f_{0}^{+}\left(\mathbf{x}_{0}, \mathbf{x}_{i}^{\prime}, t\right) d^{2} \mathbf{x}_{0} \\
& +\int_{\partial D_{0}} G_{0}^{-}\left(\mathbf{x}_{i}, \mathbf{x}_{0}, t\right) * f_{1}^{+}\left(\mathbf{x}_{0}, \mathbf{x}_{i}^{\prime}, t\right) d^{2} \mathbf{x}_{0}
\end{aligned}
$$

In Equation (12), $\int_{\partial D_{0}} G_{0}^{-}\left(\mathbf{x}_{i}, \mathbf{x}_{0}, t\right) * f_{0}^{+}\left(\mathbf{x}_{0}, \mathbf{x}_{i}^{\prime}, t\right) d^{2} \mathbf{x}_{0}$ contains primary reflections and internal multiple reflections from the overburden, $\int_{\partial D_{0}} G_{1}^{-}\left(\mathbf{x}_{i}, \mathbf{x}_{0}, t\right) * f_{0}^{+}\left(\mathbf{x}_{0}, \mathbf{x}_{i}^{\prime}, t\right) d^{2} \mathbf{x}_{0}$ and $\int_{\partial D_{0}} G_{0}^{-}\left(\mathbf{x}_{i}, \mathbf{x}_{0}, t\right) * f_{1}^{+}\left(\mathbf{x}_{0}, \mathbf{x}_{i}^{\prime}, t\right) d^{2} \mathbf{x}_{0}$ contain the counterevents of internal multiples reflections from the overburden, but with incorrect amplitudes. The term $G_{1}^{-}\left(\mathbf{x}_{i}, \mathbf{x}_{0}, t\right)$ * $f_{1}^{+}\left(\mathbf{x}_{0}, \mathbf{x}_{i}^{\prime}, t\right)$ was excluded in Equation (12), as it can degrade the quality of the data and is responsible only for multiple events of weaker amplitudes. By using only these three wavefields in Equation (12), we can apply an adaptive subtraction to remove the multiple events from the overburden that is present in $G_{0}^{-}\left(\mathbf{x}_{i}, \mathbf{x}_{0}, t\right) * f_{0}^{+}\left(\mathbf{x}_{0}, \mathbf{x}_{i}^{\prime}, t\right)$, allowing us to obtain a redatumed data without the effects of the overburden.

The main advantage of this method is that it does not require inversion, getting rid of the problem of instability, especially when using real data. Additionally, this method is computationally cheap, easy to implement and its algorithm can be parallelized in pairs of focal points (Staring et al., 2018), however, some interactions with the overburden will remain, for example, the internal multiples located between reflectors below and above the redatuming level.

\section{LSMME vs DF}

Both afore-described methods can be used as a preprocessing flow to improve the quality of an RTM image, however, there are some differences between their outputs. Figure 1 shows the difference between the outputs of both methods. The redatuming sources and receivers to a level closer to the target area with DFM can eliminate the multiples generated by the structures above that region. On the other hand, the LSMME method can eliminate the internal multiples of all orders, but still keeping the data on the surface.

When comparing the RTM images produced by migrating the outputs of these methods, it is expected that the LSMME image has less noise from the multiple reflections, since that in the DFM the multiple generated in the target area will still be present in the data as shown in Figure 1. However, the LSMME scheme needs to be evaluated for each sample in time which makes the LSMME more expensive than the DFM which is evaluated only for a single new datum (Figure 1). In the next section, we give a numerical example to validate the effectiveness and to clarify the difference between both schemes.

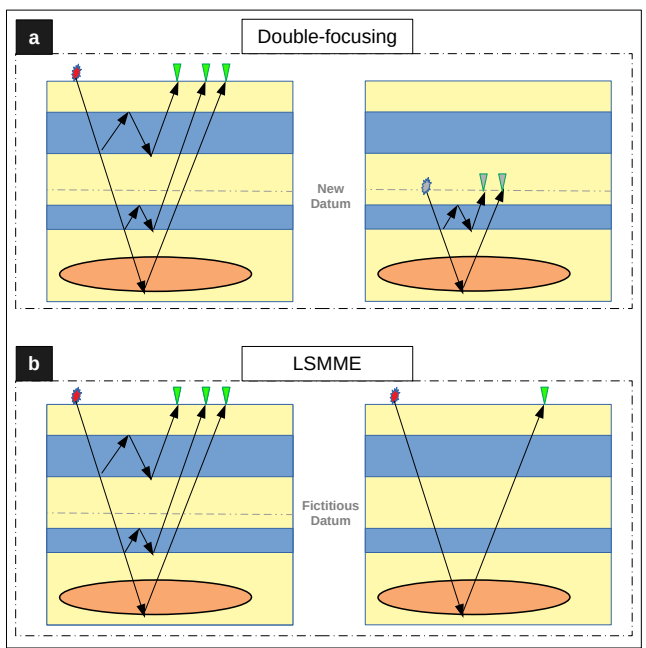

Figure 1: llustration of (a) double-focusing and (b) LSMME. Rays indicate the recorded reflection response before and after the application of both methods.

\section{Numerical example}

To validate this study, we selected a simple numerical example that allows us to make a clear interpretation of all events and also analyze the differences, advantages and disadvantages of both schemes. So, we used a model that is composed of seven flat-layers (Figure 2). This model consists of $881 \times 2001$ grid points with a grid spacing of $2.5 \mathrm{~m}$. We have computed the synthetic acoustic impulse reflection responses, for a fixed-spread geometry, with the finite-difference time-domain modeling code (Thorbecke and Draganov, 2011), and the input source signature is approximately a sinc function with a flat spectrum. We apply absorbing boundaries on all sides, i.e., we assume that surface-related multiples and ghost wave effects are removed from the recorded dataset. The direct arrival is removed from the seismic data by subtracting the simulated dataset with a homogeneous model (values of the first 
layer). The 501 sources are excited one by one and a fixed-spread array of 501 receivers with a spacing of $10 \mathrm{~m}$ is located at the top of the model. The length of each shot record is $3.204 \mathrm{~s}$ sampled at $4 \mathrm{~ms}$. To apply the LSMME, the synthetic acoustic reflection responses result from the convolution of $R$ with a Ricker source wavelet with a central frequency of $20 \mathrm{~Hz}$. It is important to add that the operators $\mathbf{R}$ and $\mathbf{R}^{*}$ are applied by using the impulse reflection responses. To apply the DFM, the direct wave of the downgoing focusing function (or direct arrival of the transmission response) $f_{0}^{+}$is estimated from a smooth velocity model by using a finite-difference solver with a Ricker source wavelet with a central frequency of $35 \mathrm{~Hz}$, then, after the subtraction, we deconvolve the redatumed data using the autoconvolution of the source, then we convolve with a Ricker wavelet with central frequency of $20 \mathrm{~Hz}$. Figure 3a shows the central shot (red star in Figure 2) of the single-sided reflection response, where we can clearly see the internal multiple reflections indicated by the red arrows.
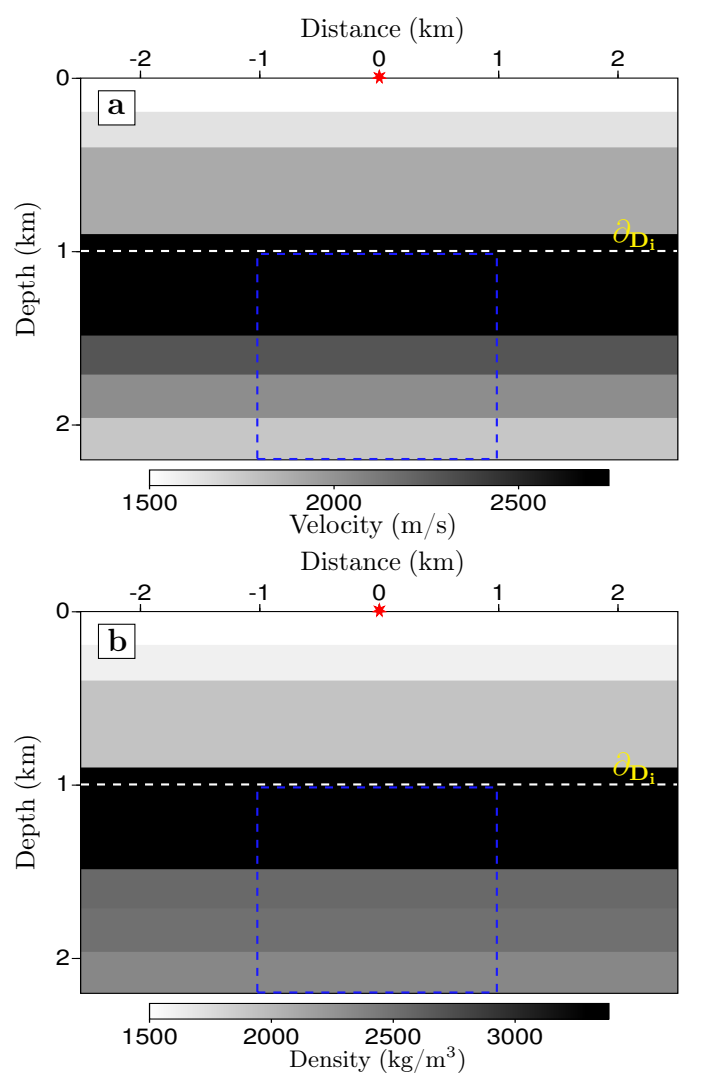

Figure 2: Flat-layers model (a) velocity and (b) density model. The blue dashed line indicates the area for imaging and the white line show the redatuming level.

The computed single-sided impulse reflection responses and the central shot (indicated by the red star in Figure 2) are used as inputs for the LSMME technique with 30 iterations. Figure $3 \mathrm{~b}$ shows the retrieved reflection response without internal multiples after applying the LSMME for the central shot gather. Figure 4 gives a comparison between the traces indicated in Figure 3 by red and black lines, where we can see that the LSMME scheme correctly eliminate the events associated with internal multiple reflections and that the primary reflections coincide well, preserving the amplitude and phase.

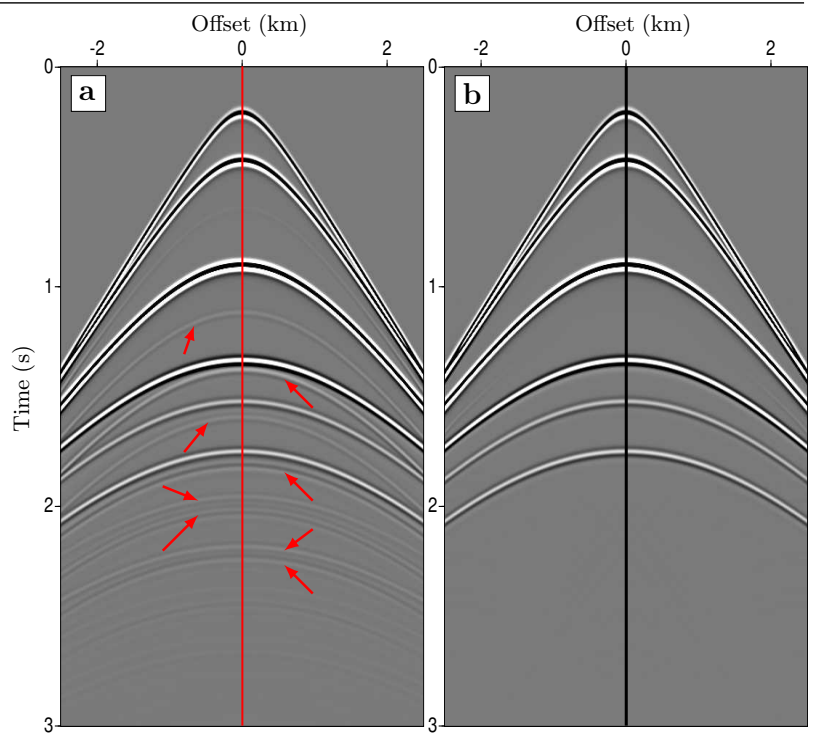

Figure 3: (a) The modeled reflection response and (b) the retrieved reflection response by the proposed LSMME.

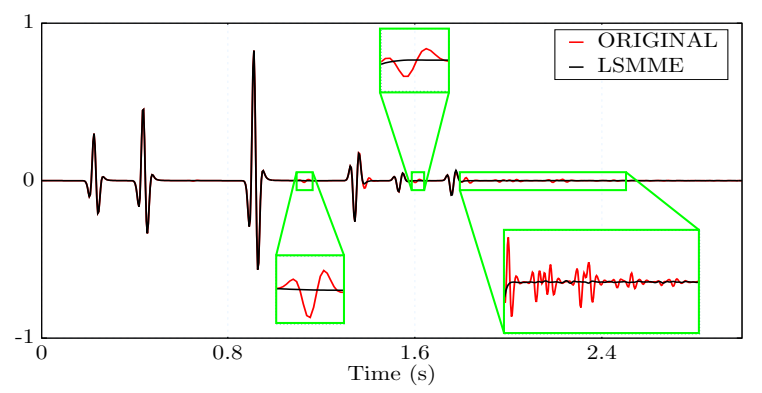

Figure 4: The comparison between the zero-offset traces from panels in Figure 3.

To use the DFM schemes we defined a depth around $1000 \mathrm{~m}$ as the new datum to redatuming (as indicate the white line, outlined in Figure 2). After obtaining the wavefield containing primaries and multiples, $G_{0}^{-} * f_{0}^{+}$, and the wavefields that contain the internal multiples from overburden, $G_{0}^{-} * f_{1}^{+}$and $G_{1}^{-} * f_{0}^{+}$, we applied the adaptive subtraction using a regularized nonstationary regression (Fomel, 2009). Figure 5b shows the redatumed reflection response after applying a muting that removes the acausal amplitudes and the direct wave. The effectiveness of the DFM method can be confirmed by comparing it with the modeled reflection response (Figure $5 \mathrm{a}$ ) without the direct wave, obtained in a medium that is homogenous above the redatuming level such that the overburden does not exist. From this result, we can see that the reflection response obtained by the adaptive double-focusing method resembles the modeled reflection response, while still retaining some higher order multiple events from the overburden, with a lower amplitude, as indicated by the red arrows in Figure 5b.

The comparison of LSMME with DFM is made in the image domain. So, after the application of both schemes in the dataset, we generated depth images using reverse-time migration. We used the original dataset, the retrieved reflection responses by LSMME and the data redatumed by DFM as inputs to image the target zone (see the blue rectangle in Figure 2). A comparison between the migrated 


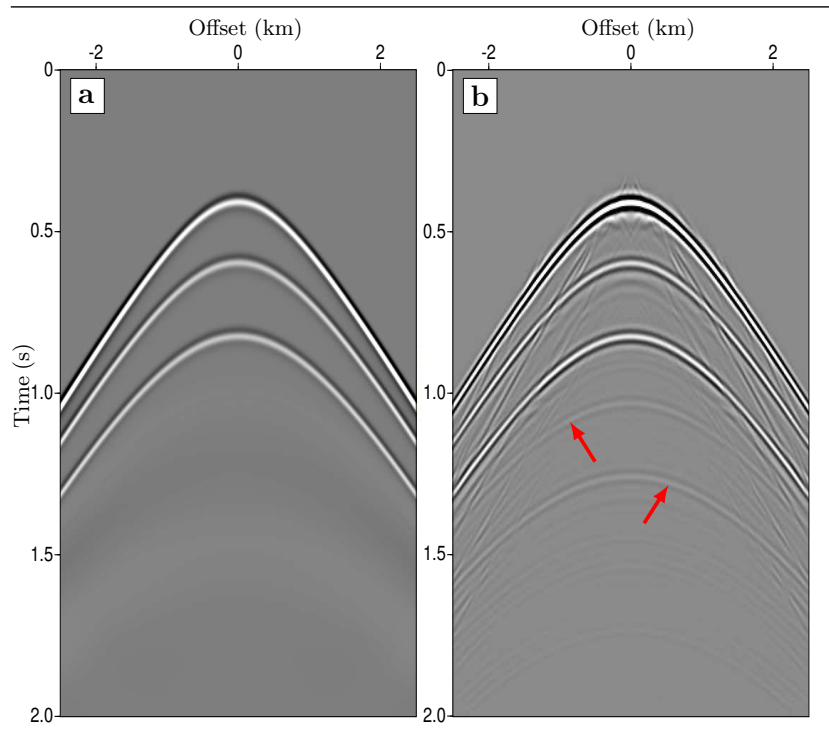

Figure 5: (a) Redatumed reflection response obtained by modeling in a medium that is homogeneous above the redatuming level and (b) the result of the adaptive doublefocusing method.

images is shown in Figure 6. The image of the original dataset in Figure 6a contains artifacts from internal multiple reflections because they are imaged as if they were primary reflections (indicated by the red arrows). In Figure 6b, we see that the RTM image from the DFM dataset is free internal multiples generated in the overburden as indicated by the yellow arrow, and some artifacts are indicated by the red arrows. The image of the retrieved LSMME dataset shown in Figure $6 c$ is free of all artifacts caused by the internal multiples.

Both methods were run using 4 nodes configured with 376 GB of RAM (DDR4) memory, 36 Intel(R) Xeon(R) Gold 6240 CPU @ 260GHz. While LSMME took a total of 9.5 hours in the filtering process, the DFM spent about 3 minutes to obtain the redatumed data plus 1 hour to perform the adaptive subtraction.

\section{Discussions}

When we analyze the effects of LSMME in comparison with the original dataset in Figures 3 and 4, we realize that this method is efficient in removing multiples of all orders while preserving primary reflections. The image generated with the data filtered by the LSMME (Figure 6c) is free of the false artifacts present in the image generated with the original data (Figure 6a).

The comparison of the DFM with the reflection response modeled at the redatuming level (Figure 5) shows that the DFM scheme is able to reposition the reflectors at the desired datum. However, in Figure $5 b$ remains some overburden events of order higher than one (indicated by the red arrows) that are not present in Figure $5 \mathrm{a}$. Additionally, there are still some artifacts that could be removed by a more effective adaptive subtraction method. Figure $6 \mathrm{~b}$ shows the depth image obtained by the RTM of the DFM dataset. This image is now free of the artifact that is generated by the multiple of the overburden (yellow arrow) as indicated in the RTM image of the original dataset

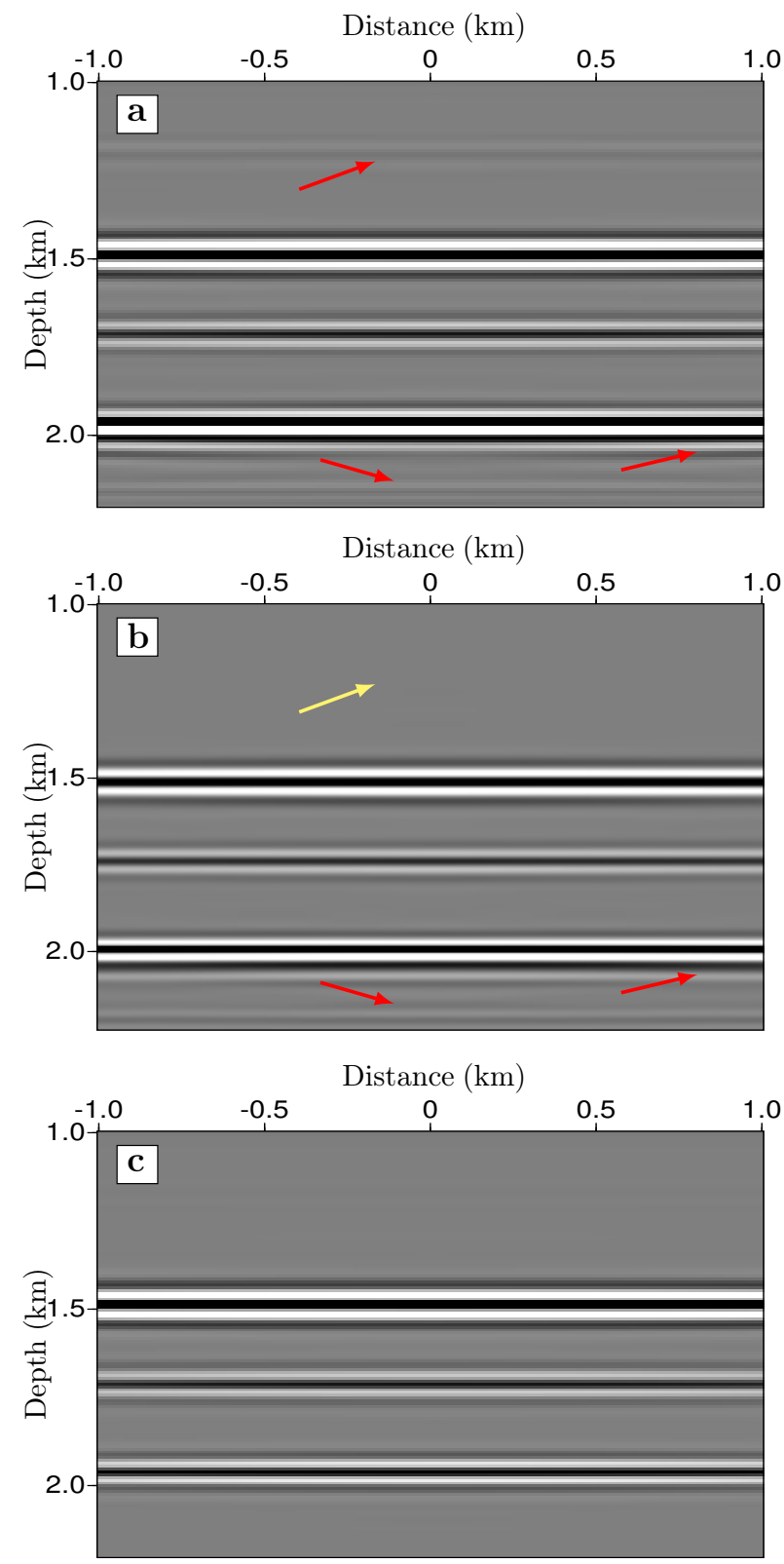

Figure 6: RTM images with (a) complete field data at the surface, (b) after applying DFM, and (c) after LSMME.

(Figure 6a). However, other artifacts remain (signaled by the red arrows) which is in accordance with redatumed data. By comparing the images generated using DFM and LSMME data as inputs (Figures $6 \mathrm{~b}$ and $6 \mathrm{c}$, respectively), we can observe that the LSMME image is absent from all artifacts caused by multiple internal reflections, while in the DFM image there are still artifacts caused by some events of multiple reflections, showing that the LSMME is more efficient on the removal of internal multiples than DFM.

These results show the advantages and disadvantages of both these schemes. We can cite as an advantage of LSMME the fact that it eliminates or attenuates multiple reflections without using a velocity model or adaptive filter in a fully automated and data-driven and can provide a cleaner image. While the DFM has these two prerequisites, it runs at a significantly lower computational cost, and as a redatuming method it can have broader 
applications other than imaging.

\section{Conclusions}

In this paper, we compare two strategies to treat the internal multiple reflections in a simple numerical example, and analyze their limitations, disadvantages and advantages of both. The results showed that the doublefocusing method is robust for eliminating the main multiple events generated for the overburden at a low computational cost. However, its effectiveness depends on the chosen adaptive subtraction method. On the other hand, we saw that the LSMME is more robust than the DFM in the treatment of internal multiple reflections, being able to eliminate multiples of all orders present in the dataset. The great disadvantage of LSMME is its high computational cost, but this can be mitigated with the use of highperformance computing. We conclude that if a good adaptive filter and a correct velocity model are available, the use of DFM will be enough to eliminate most of the multiples of the overburden. Therefore, this could provide an image of high quality in the target area. However, if computational resources are available and the goal is to obtain a cleanest possible image, free of all the effects of internal multiples in the dataset in a fully automated process, independent of velocity information or adaptive filter, then LSMME is the more suitable choice.

\section{ACKNOWLEDGMENTS}

This work was supported by CENPES/Petrobras through the Marchenko project at SENAI CIMATEC. The authors also wish to thank to the developer team of the OpenSource libraries in TUDelft. A special acknowledgment to the geophysical team at SENAI CIMATEC for discussions and contributions.

\section{References}

Fomel, S., 2009, Adaptive multiple subtraction using regularized nonstationary regression: Geophysics, 74, V25-V33.

Paige, C. and M. Saunders, 1982, Algorithm 583. LSQR: Sparse linear equations and least squares problems: ACM Transactions on Mathematical Software, 8, 195209.

Santos, R. S., D. E. Revelo, R. C. Pestana, V. Koehne, D. F. Barrera, and M. S. Souza, 2020a, A least-squares based approach for the marchenko internal multiple elimination scheme, in SEG Technical Program Expanded Abstracts 2020, 3184-3188, Society of Exploration Geophysicists.

Santos, R. S., M. S. Souza, D. F. Barrera, D. E. Revelo, and V. Koehne, 2020b, Fast marchenko multiples elimination on $\mathrm{cmp}$ processing, in SEG Technical Program Expanded Abstracts 2020, 3189-3193, Society of Exploration Geophysicists.

Staring, M., R. Pereira, H. Douma, J. van der Neut, and K. Wapenaar, 2018, Source-receiver marchenko redatuming on field data using an adaptive doublefocusing method: Geophysics, 83, S579-S590.

Staring, M. and K. Wapenaar, 2020, Three-dimensional marchenko internal multiple attenuation on narrow azimuth streamer data of the santos basin, brazil:
Geophysical Prospecting, 68, 1864-1877.

Thorbecke, J. and D. Draganov, 2011, Finite-difference modeling experiments for seismic interferometry: Geophysics, 76, $\mathrm{H} 1-\mathrm{H} 18$.

van der Neut, J., J. Thorbecke, K. Mehta, E. Slob, and K. Wapenaar, 2011, Controlled-source interferometric redatuming by crosscorrelation and multidimensional deconvolution in elastic media: Geophysics, 76, SA63SA76.

van der Neut, J. and K. Wapenaar, 2016, Adaptive overburden elimination with the multidimensional Marchenko equation: Geophysics, 81, T265-T284.

Wapenaar, K., J. Brackenhoff, M. Dukalski, G. Meles, C. Reinicke, E. Slob, M. Staring, J. Thorbecke, J. van der Neut, and L. Zhang, 2021, Marchenko redatuming, imaging and multiple elimination, and their mutual relations: Geophysics, 86, 1-103.

Zhang, L. and E. Slob, 2019a, A field data example of Marchenko multiple elimination: Geophysics, 85, 1-39.

- 2019b, Free-surface and internal multiple elimination in one step without adaptive subtraction: Geophysics, 84, A7-A11.

_ 2020a, A fast algorithm for multiple elimination and transmission compensation in primary reflections: Geophysical Journal International, 221, 371-377.

- 2020b, Marchenko multiple elimination of a laboratory example: Geophysical Journal International, 221, 1138-1144.

Zhang, L. and M. Staring, 2018, Marchenko scheme based internal multiple reflection elimination in acoustic wavefield: Journal of Applied Geophysics, 159, 429433.

Zhang, L., J. Thorbecke, K. Wapenaar, and E. Slob, 2019, Transmission compensated primary reflection retrieval in the data domain and consequences for imaging: Geophysics, 84, Q27-Q36. 